\title{
Numerical Simulation Analysis on Sailing Resistance of Amphibious Vehicle
}

\author{
Wenfeng Guo ${ }^{1, a}$, Yutian Pan ${ }^{2, b}$ and CunZhen Xing ${ }^{3, c}$ \\ ${ }^{1}$ School of Equipment and Engineering, Shenyang Ligong University, Shenyang, China \\ ${ }^{2}$ School of Mechatronics Engineering, North University of China, Taiyuan, China \\ ${ }^{3}$ Liaoshen Industrial Group Co., Ltd. Shenyang, China \\ a shirly-guo@139.com, bpanyutiannuc@163.com, xingczhen318@163.com
}

Keywords: amphibious vehicle; sailing resistance; simulation; towing test

\begin{abstract}
Through numerical simulation method based the Fluent to find the rule of the sailing resistance of the amphibious vehicle in calm water. By comparing the results of the simulation and the towing test, it is observed that the simulation results is accorded very well with the test results .It is proved that the simulation can show the real performance of the vehicle, and which is a reliable data for the resistance reduction and speed increase research of the amphibious vehicle.
\end{abstract}

\section{Introduction}

The main factors influencing the vehicle 's speedability are sailing resistance and propulsion performance. Empirically, the study of resistance was usually reference to the ship theory. But, the ship theory can't express the change of the resistance accurately because of the complexity of shape structure of amphibious vehicle. In recent years, computational fluid dynamics has become an effective tool for the study of complex flow problems, and develop continuously. According to the situation of less research about amphibious wheeled vehicle performance on water, so this paper pursue the research on the numerical simulation of flow field characteristics based on FLUENT, simulate navigation process and get the resistance law of amphibious vehicle by use of computational fluid dynamics software ,analyze dynamic characteristics, providing a reference basis for the study of resistance reduction and speed increase of amphibious vehicle.

\section{The Research Object}

This paper took an $8 * 8$ amphibious vehicle as the research object, modeled based on ANSYS WORKBENCH, the proportion of the simulation model was 1:1, the accessories model was simplified with breakwater plate.

\section{The Control Equation}

The calculation about flow around of amphibious vehicles satisfied the continuity equation, momentum equation and $\mathrm{k}-\varepsilon$ turbulence model.

The continuity equation:

$$
\begin{aligned}
& \frac{\partial \alpha_{\alpha}}{\partial t}+\vec{V} \cdot \nabla \alpha_{\alpha}=0 \\
& \frac{\partial \alpha_{w}}{\partial t}+\vec{V} \cdot \nabla \alpha_{w}=0
\end{aligned}
$$

The momentum equation:

$$
\frac{\partial}{\partial t}(\rho \vec{V})+\nabla \cdot(\rho \vec{V} \vec{V})=-\nabla p+\nabla\left[\mu\left(\nabla \vec{V}+\nabla \vec{V}^{T}\right)\right]+\rho \vec{g}+\vec{F}
$$

In the standard $\mathrm{k}-\varepsilon$ turbulence model, $\mathrm{k}$ and $\varepsilon$ are the two basic unknown quantity, and the corresponding transport equation: 


$$
\begin{aligned}
& \frac{\partial}{\partial t}(\rho k)+\nabla \cdot(\rho k \vec{V})=\nabla\left[\left(\mu+\frac{\mu_{t}}{\sigma_{k}}\right) \nabla k\right]+G_{k}-\rho \varepsilon \\
& \frac{\partial}{\partial t}(\rho \varepsilon)+\nabla \cdot(\rho \varepsilon \vec{V})=\nabla\left[\left(\mu+\frac{\mu_{t}}{\sigma_{k}}\right) \nabla \varepsilon\right]+\rho C_{1} S \varepsilon-\rho C_{2} \frac{\varepsilon^{2}}{k+\sqrt{v \varepsilon}}
\end{aligned}
$$

of which: $\mu$--dynamic viscosity coefficient; $\mu_{\mathrm{t}^{--}}$turbulent viscosity coefficient; Gk-- generation of the turbulent kinetic energy $\mathrm{k}$ caused by the mean velocity gradient ; $\sigma_{\mathrm{k}}$ and $\sigma_{\mathrm{z}}$-- the turbulent

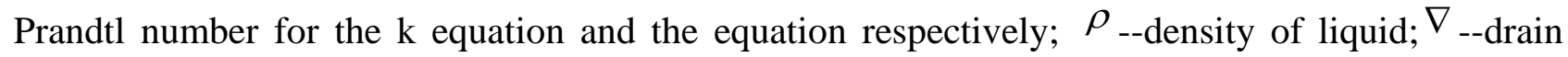
volume; $C_{2}$-- constant; ${ }^{v}$--Kinematic viscosity coefficient.

The turbulent viscosity coefficient $\mu_{\mathrm{t}}$ can be expressed as functions of $k$ and $\varepsilon$,

$$
\mu_{t}=\rho f_{u} C_{\mu} \frac{k^{2}}{\varepsilon} \text {. }
$$

In this paper, any phase of all satisfy the continuity, water was considered to be incompressible Newton fluid, in addition, the air can also be used as incompressible fluid because of the low speed of the amphibious vehicle. The speed is including three component as $u, v, w$. Assume that the volume ratio of water and air which occupies in the unit is $\alpha_{\mathrm{w}}$ and $\alpha_{\alpha}$ respectively, their density and viscosity were $\rho_{\mathrm{w}}, \mu_{\mathrm{w}}, \rho_{\alpha}, \mu_{\alpha}$. Of which: The sum of the volume fraction of water or air in each control unit should be 1 :

$$
\alpha_{w}+\alpha_{\alpha}=1
$$

\section{The flow field boundary condition setting}

According to the requirement of the amphibious vehicle speed, and calculation is carried in different flow spatial ,it is found that significant effect to the body just appeared in limited areas around, so the flow field is choose the regional of $50 \mathrm{~m} * 30 \mathrm{~m} * 15 \mathrm{~m}$.Entrance conditions: Air and water entrance was that the speed entrance, the entrance flow velocity as the vehicle's navigation speed, It is not obvious that the influence of turbulence intensity on the stream surface which to the body resistance .Exit conditions: The surface far behind the body was the outflow boundary which is owned the free outflow boundary condition because the pressure and velocity on the out flow boundary are unknown, and the exit condition is confirmed by FLUENT internal calculation. Wall condition: The vehicle's surface is as the solid wall boundary condition. The symmetric boundary: The vehicle model mid-ship section extend face was defined as the symmetric boundary which can make the solution scale to half the entire problem. Free liquid surface condition: The computational domain was divided into two parts of air and water by the free liquid surface position according to the balance at the initial state, and given the uniform velocity. Other initial conditions: The density and viscosity of water and air were the values at $12 \mathrm{C}$, environmental pressure was a standard atmospheric pressure, acceleration of gravity was $9.81 \mathrm{~m} / \mathrm{s}^{2}$.

\section{Meshing}

In this paper, the meshes division of vehicle body and its flow field had been done based on the WORKBENCH software. The vehicle model was closed with a small rectangular, using strong adaptability hybrid-unstructured meshes for the grid division. The total number of the model grids is 830000 under the water speed of $9 \mathrm{~km} / \mathrm{h}$. The mesh quality is good with self-checking, and it can guarantee the of calculation convergence and accuracy of solution. Then mesh file was imported into the FLUENT software, and starting the simulation calculation.

\section{Calculation Results Analysis}

As shown in Tab.1,the sailing resistance under several kinds of different speed has been got 
through the simulation software, from the results that the resistance was increasing with the sailing speed increased. The greater the speed, the greater resistance value of the body is.

Tab.1 The simulation results of sailing resistance

\begin{tabular}{cccccccc}
\hline Velocity[km/h] & 3 & 4 & 5 & 6 & 7 & 8 & 9 \\
\hline Resistance[KN] & 0.691 & 1.317 & 2.353 & 3.974 & 5.079 & 6.026 & 7.15
\end{tabular}

Fig. 1 shows the vehicle dynamic pressure diagram. From the map, which should be observed that the maximum pressure value occurred at the junction of lower part of the tail and of the car ,this is due to the blocking which caused by water flow at the vehicle body curvature change. The dynamic pressure value was relatively large on the front wheels, this is because of the larger frictional resistance caused by the wheels irregular profile. A low pressure are occurred at the gap of the wheels, and accompanied with the eddy current, the effect on the resistance of amphibious vehicle was remarkable.
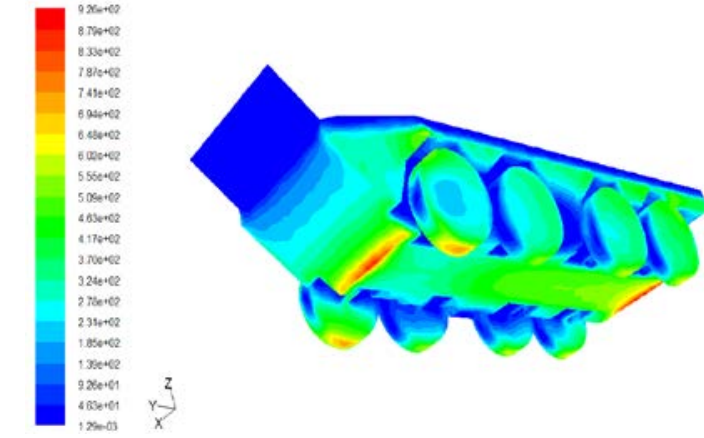

Fig. 1 The dynamic pressure distribution picture of the vehicle body

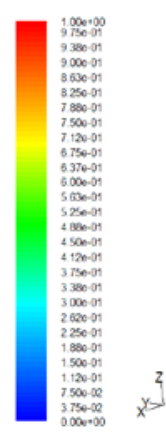

Fig. 2 The aqueous phase distribution picture of the vehicle body

The Fig.2 is water distribution map, blue represents air, the red part represents the water. The waves was stirred up by the breakwater pushing water, and caused water on the car first and then produced the wave resistance. When the speed is higher, the wave resistance value of the proportion will become larger. The breakwater's inflow angle size affect the dynamic pressure function of which the fluid wave to vehicle body .So the inflow angle of the breakwater plate must be one of the factors that can reduce the sailing resistance which will be considered.

\section{Towing trial test}

In order to verify the accuracy of numerical simulation, the towing resistance test was carried out. The real vehicle's sailing resistance was obtained by resistance conversion based on the experimental observations. The experiment was carried out under 7 kinds of speed, and the results both of towing test and simulation were plotted to form the comparison curves as shown as Fig.5.According to the comparison curves under design speed, it can be found that the two curves trend was consistent relatively beside of a little error. The two curves were conforming with the resistance change rule of displacement amphibious vehicle. So the numerical simulation method can be used as the main research method of resistance research of amphibious vehicle. 


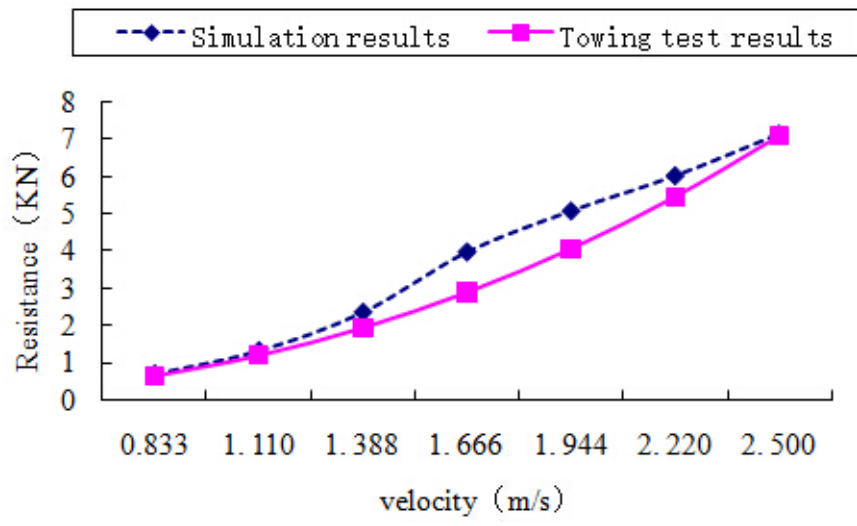

Fig.3 The comparison curves of both test and simulation results

\section{Conclusion}

The numerical simulation method based on FLUENT for wheeled amphibious vehicle research on resistance is feasible, and with high reliability for the simulation results.

Through the comparison of numerical simulation and towing test results, it is obtained that the sailing resistance of the wheeled amphibious vehicle rised proportionately to sailing speed's power .it accorded well with the resistance change rule of displacement amphibious vehicle.

\section{Reference}

[1] Song Guixia,ZhaoYouqun.3-D numerical simulation on resistances of amphibious vehicles using tow-phase flow. Journal of Harbin Engineering University.9(2008)907 911.

[2] Wang Tao, Xu Guoying, Amphibious vehicle water dynamic performance numerical simulation method and application.Beijing,2009.

[3] Li Yuliang, Pan Shuangxia, Volume of fluid model based parallel numerical computation of resistance for amphibious vehicles.Journal of Zhejiang University (Engineering Science). 8 (2006)1333 1338.

[4] Ni Chongben, A comprehensive investigation of ship resistance prediction based on CFD theory. Shanghai, 2011. 